\title{
Toward a general theory of evolution: Extending Darwinian theory to inanimate matter
}

Addy Pross

\begin{abstract}
Though Darwinian theory dramatically revolutionized biological understanding, its strictly biological focus has resulted in a widening conceptual gulf between the biological and physical sciences. In this paper we strive to extend and reformulate Darwinian theory in physicochemical terms so it can accommodate both animate and inanimate systems, thereby helping to bridge this scientific divide. The extended formulation is based on the recently proposed concept of dynamic kinetic stability and data from the newly emerging area of systems chemistry. The analysis leads us to conclude that abiogenesis and evolution, rather than manifesting two discrete stages in the emergence of complex life, actually constitute one single physicochemical process. Based on that proposed unification, the extended theory offers some additional insights into life's unique characteristics, as well as added means for addressing the three central questions of biology: what is life, how did it emerge, and how would one make it?
\end{abstract}

\section{Introduction}

Despite the enormous developments in molecular biology during the past half century, the science of biology appears to have reached a conceptual impasse. Woese [1] captured both the nature and the magnitude of the problem with his comment: "Biology today is no more fully understood in principle than physics was a century or so ago. In both cases the guiding vision has (or had) reached its end, and in both, a new, deeper, more invigorating representation of reality is (or was) called for." The issue raised by Woese is a fundamental one - to understand the genesis and nature of biological organization and to address biology's holistic, rather than just its molecular nature. Kauffman [2] expressed the difficulty in somewhat different terms: "....we know many of the parts and many of the processes. But what makes a cell alive is still not clear to us. The center is still mysterious." In effect, the provocative question, "What is Life?", raised by Schrödinger over half a century ago [3], remains unresolved, a source of unending debate. Thus, despite the recent dramatic insights into the molecular character of living systems, biology of the $21^{\text {st }}$ century is continuing to struggle with the very essence of biological reality.

Correspondence: pross@bgu.ac.il

Department of Chemistry, Ben Gurion University of the Negev, Be'er Sheva, 84105, Israel
At the heart of biology's crisis of identity lies its problematic relationship with the two sciences that deal with inanimate matter - physics and chemistry. While the on-going debate regarding the role of reductionist thinking in biology exemplifies the difficulties at a methodological level, the problematic relationship manifests itself beyond issues of methodology and philosophy of science. Indeed, the answers to two fundamental questions, central to understanding the life issue, remain frustratingly out of reach. First, how did life emerge, and, second, how would one go about synthesizing a simple living system? Biology cannot avoid these questions because, together with the 'what is life?' question, they form the three apexes of the triangle of holistic understanding. Being able to adequately answer any one of the questions depends on being able to answer the other two. A coherent strategy for the synthesis of a living system is not possible if one does not know what life is, and one cannot know what life is if one does not understand the principles governing its emergence. Richard Feynman's aphorism (quoted in [4]) captured the issue succinctly: "What I cannot create, I do not understand". Remarkably, the laws of physics and chemistry, the two sciences that deal with material structure and reactivity, have as yet been unable to adequately bridge between the physicochemical and biological worlds. 
Despite the above-mentioned difficulties we believe that the problem is resolvable, at least in principle. If the widely held view that life did emerge from inanimate matter is correct, it suggests that the integration of animate and inanimate matter within a single conceptual framework is an achievable goal. This is true regardless of our knowledge of the detailed historical path that led from inanimate to animate. The very existence of such a pathway would be proof for that. If indeed such a conversion did take place, it suggests that particular laws of physics and chemistry, whether currently known or not, must have facilitated that transformation, and therefore those laws, together with the materials on which they operated, can form the basis for understanding the relationship between these two fundamentally distinct material forms.

In this paper we wish to build on this way of thinking and to draw the outlines of a general theory of evolution, a theory that remains firmly rooted in the Darwinian landscape, but reformulated in physicochemical terms so as to encompass both biological and non-biological systems. Such a theory, first and foremost, rests on a basic assumption: that the physicochemical principles responsible for abiogenesis, the so-called chemical phase - the stage in which inanimate matter complexified into a simple living system - are fundamentally the same as those responsible for biological evolution, though for the biological phase these principles are necessarily dressed up in biological garb. Darwin would no doubt have drawn enormous satisfaction from such a proposal, one that attempts to integrate Darwinian-type thinking into the physicochemical world. However such a sweeping assumption needs to be substantiated. Accordingly our analysis is divided into two parts. In the first part we argue for the basis of that assumption, and in second part we attempt to describe key elements of that general theory, as well as the insights that derive from it, in particular with regard to the three central questions of biology, referred to above. The analysis draws heavily on data from the emergent research area termed by Günter von Kiedrowski, 'Systems Chemistry' [5,6]. The essence of this emergent area is to fill the chemical void between chemistry and biology by seeking the chemical origins of biological organization.

\section{Discussion}

\subsection{Unification of abiogenesis and evolution}

Darwinian theory lies at the very heart of modern biology, and rightly so. As Dobzhansky [7] famously noted: "Nothing in biology makes sense except in the light of evolution". Yet despite the extraordinary and overwhelming impact of Darwin's ideas on biology and beyond, Darwinian theory does not address the Origin of Life problem, even though the nature and source of early life could well impact on the theory itself. Interestingly, this limitation was already obvious to Darwin's contemporaries. Thus just three years after the publication of Darwin's monumental thesis, Haeckel $[8,9]$ pointed out that "the chief defect of the Darwinian theory is that it throws no light on the origin of the primitive organism-probably a simple cell-from which all the others have descended. When Darwin assumes a special creative act for this first species, he is not consistent, and, I think, not quite sincere...". Surprisingly, this early concern seems to have dissipated with time. Thus a leading biologist, Richard Dawkins, in the opening line of his book 'The Blind Watchmaker' writes: “... our existence once presented the greatest of all mysteries, but that it is a mystery no longer because it is solved. Darwin and Wallace solved it...." [10]. Yes, Darwinism did resolve the dilemma of how microscopic complexity was transformed into macroscopic complexity, however it did not resolve or even address the most vexing of questions: how did the extraordinary microscopic complexity of the simplest living system emerge in the first place?

It is not surprising then that the emergence of complex life on earth is divided into two phases - abiogenesis, the chemical phase, and evolution, the biological phase (as illustrated in 1), with the first phase being the one that remains a source of confusion and continuing controversy (for a recent special issue on the origin of life, see [11], for general reviews, see [12-15]). But what if these two stages could be conceptually merged into one single continuous physicochemical process? Such unification could significantly impact on our understanding of the life phenomenon, as we would then be faced with the need to understand just one single process, rather than two separate and discrete processes. And given our broad understanding of the Darwinian phase, that understanding could be immediately applied to the poorly understood earlier chemical phase. But are there reasonable grounds for such a sweeping proposal? We believe the answer to be yes, and base this view on recent developments in systems chemistry. Recent work on molecular replicating systems has revealed that several phenomena associated with replicating chemical systems are also manifest in biological systems. This general observation is crucially important because it provides the empirical basis for the conceptual link

\begin{tabular}{|c|c|c|}
\hline \multirow{2}{*}{ Non-Life } & $\begin{array}{c}\text { Chemical } \\
\text { phase }\end{array}$ & $\begin{array}{c}\text { Biological } \\
\text { phase }\end{array}$ \\
\hline & Life & $\begin{array}{l}\text { Darwinian } \\
\text { theory }\end{array}$ \\
\hline $\begin{array}{l}\text { Scheme } 1 \\
\text { non-life into }\end{array}$ & $\begin{array}{l}\text { o-phase (chemical and b } \\
\text { omplex life. }\end{array}$ & ological) transformation of \\
\hline
\end{tabular}


between chemistry and biology, not just at the self-evident structural level (i.e., both animate and inanimate matter are constructed from atomic and molecular entities), but at some deeper organizational level. Indeed this evidence will form the basis of our proposal that the chemical and biological phases are in fact one single process. Let us first review the relevant empirical data.

\subsubsection{Natural selection at the chemical level}

Already in the 1960s, Mills et al. [16] noted that a molecular replicating system, Q $\beta$ RNA, when reacted with activated nucleotides in the presence of the appropriate replicase, underwent a process of replication, mutation, selection, evolution, in striking analogy to biological systems. The RNA oligonucleotide, originally some 4200 bases in length, replicated, mutated and evolved into a much shorter oligonucleotide chain just $17 \%$ of the original length, that replicated much faster than the original chain $[15,16]$. This observation, even on its own, suggests that Darwinian behavior, a fundamentally biological phenomenon, has its roots in chemistry. No one would seriously argue that a single molecule, whatever its structure, is in any meaningful sense 'alive', yet Darwinian-type behavior is strikingly evident at this inanimate, molecular level $[15,16]$. Since then in vitro evolution procedures have been developed and extended to cover a wide range of nucleic acid systems as demonstrated by the work of Bartel and Szostak [17], Johnston et al. [18] and Joyce et al. [19,20], thereby emphasizing the generality of evolutionary-like processes at the molecular level.

An even more striking expression of natural selection at the chemical level that further highlights the extent of the chemistry-biology nexus has recently been reported by Voytek and Joyce [21]. A key ecological principle, the competitive exclusion principle [22] states: "Complete competitors cannot coexist", or in its more positive expression: "Ecological differentiation is the necessary condition for coexistence". That principle informs us that two non-interbreeding populations that occupy precisely the same ecological niche (i.e., both competing for the same resource) cannot coexist - one will drive the other into extinction. The striking aspect of Voytek and Joyce's study was that it demonstrated that the roots of this quintessential biological principle can be found in chemistry. They reported that two RNA enzymes, when allowed to replicate and evolve in the presence of an essential substrate, were unable to coexist. One of the enzymes drove the other into extinction in line with the prediction of the competitive exclusion principle. More significantly, however, when the two enzymes were simultaneously reacted with five alternative substrates, the two enzymes were able to coexist. Each RNA enzyme evolved so as to optimize its utilization of one of the 5 substrates (a different substrate for each of the two enzymes) so that the system effectively mimicked biological niche behavior, again in accord with the exclusion principle. Darwin's classic finches niche behavior [23] at the chemical level!

As noted above, both chemical and biological replicators respond in a strikingly similar way to the replication-mutation-selection-evolution causal chain. But there is an additional consequence of that causal chain that manifests itself at both the chemical and the biological levels - the process of complexification. Let us explain.

\subsubsection{Complexification at both chemical and biological levels}

Within the biological world there is no doubting that a definite process of complexification over the extended evolutionary time frame has taken place. Though the detailed path toward cellular complexity remains controversial, the existence of that evolutionary drive toward greater complexity cannot be denied. Thus it is conventional wisdom to believe that the more complex eukaryotic cell evolved from simpler prokaryotic cellular organization, most likely following some endosymbiotic event [24], and in a more recent evolutionary saltation, that multi-cellular organisms evolved from single cell ones. The evolutionary dynamic appears to be driven, at least in part, by the biological advantages associated with complexification.

Given the unambiguous evidence for complexification during biological evolution, it is of cardinal interest to observe whether that same complexification tendency can be discerned at the chemical level, i.e., within relatively simple chemical replicating systems. In view of the relatively brief period of time such systems have been studied the amount of data remains limited. Nonetheless some preliminary conclusions may be tentatively offered. The idea of a hypercyclic cooperative network at the molecular level was first proposed by Eigen and Schuster [25], but it was only in 1994 that replication based on the cross-catalysis of two oligonucleotides was reported by Sievers and von Kiedrowski [26]. Subsequently other functional groups were also shown to exhibit autocatalytic behavior through the establishment of cooperative cross-catalytic networks. Thus Lee et al. [27] and Yao et al. [28] demonstrated network formation in self-replicating peptides and, more recently, Kindermann et al. [29] and Kassianidis and Philp [30] have observed cross-catalysis in a self-replicating Diels Alder reaction, suggesting that cooperative molecular behavior within a replicative context may be quite general.

A more explicit demonstration of the replicating advantages associated with a network as opposed to an individual molecular replicator, however, was recently demonstrated by Lincoln and Joyce [31]. Whereas a 
particular RNA autocatalyst was incapable of more than two successive doublings, each of which took about 17 $\mathrm{h}$ to occur, conversion of that RNA ribozyme into a cross-catalytic network based on two RNA ribozymes resulted in the formation of a rapidly replicating system with a doubling time of just $1 \mathrm{~h}$, which could be sustained indefinitely. Thus a cooperative cross-catalytic system derived from an autocatalytic parent through an evolutionary process, proved to be a more effective replicator ("fitter" in biological jargon), than the autocatalytic parent precursor. The above results, though still limited in scope, suggests that cooperative behavior can emerge and manifest itself at the molecular level, that the drive toward more complex replicating systems appears to underlie chemical, and not just biological, replicators. The implications of these preliminary findings appear to be far-reaching. They suggest that the biological drive toward greater complexity has its roots in chemistry, that the entire evolutionary process can be traced back to kinetic forces at the molecular level!

\subsubsection{Significance of common patterns at chemical and biological levels}

The observation of the same complexification tendency in both chemical and biological phases is significant in yet another sense. Complexification is not just a phenomenon associated with the two phases, it can also be viewed as the mechanism by which the chemical phase eventually merges with (and into) the biological phase! Ultimately the primary distinction between the chemical and biological phases appears to be in the degree of complexification that has come about, rather than in the nature of the process itself. Clearly then, the trend toward greater complexity that is manifest at the chemical level could be expected to lead over the extended evolutionary time scale to the enhanced complexity that is evident at the biological level. Thus complexification, primarily through network establishment that maintains the system's holistic replicative capability, is the means by which the simple replicating systems of chemistry are transformed over time into the highly complex replicating systems that we term biology. The implication is clear: life's emergence began with the chance appearance of some relatively simple replicating chemical system, which then began the long road toward increasingly complex replicating entities.

\subsection{Toward a general theory of evolution \\ 2.2.1. Uncovering the chemical roots of Darwinism}

We have already pointed out that the Darwinian-type thinking has been applied to molecular replicators, thereby extending its reach to the chemical domain. However a methodological difficulty arises with this approach. Consider, Darwinian theory was proposed on the basis of data, terminology, and concepts that are all biological. Darwinian theory is therefore, by definition, a biological theory. Indeed, being a biological theory, Darwin himself considered the possibility of an earlier chemical phase preceding the biological phase as one that could not be adequately addressed within that biological framework. In a now famous letter to Joseph Dalton Hooker written in March 1863, Darwin wrote: “...it is mere rubbish thinking at present of origin of life; one might as well think of origin of matter" [9]. Accordingly, if the chemical and biological phases constitute a single physicochemical process as we have suggested, then it logically follows that Darwinian theory needs to be extended and reformulated so that it can also encompass inanimate chemical systems. Note that it is not sufficient to simply conclude that Darwinian concepts are applicable to chemical systems as well as biological ones. While not denying the didactic value of such thinking, the application of biological concepts to chemical phenomena is, in a scientific methodological sense, problematic, even flawed. Deeper insights into the biological-chemical connection can be provided, but only when the connection is approached in the reverse direction. Let us elaborate on this key idea.

Scientific reductionism, a central scientific methodology, teaches us to seek understanding within higher hierarchical level sciences by using concepts from lower hierarchical level sciences, not the other way around. That suggests we should seek to explain biological phenomena in chemical terms, not chemical phenomena in biological terms. To clarify the point with an extreme example, consider the two sciences, chemistry and psychology. While a proposed molecular explanation for some psychological phenomenon might be intriguing and arouse interest, a psychological explanation for some molecular phenomenon would only be met with derision! To quote Weinberg [32]: "Explanatory arrows always point downward". Thus we routinely attempt to explain psychological phenomena in biological terms, biological phenomena in physical and chemical terms, chemical phenomena in physical terms, and so on, not the other way around. The observation of Darwinian-like behavior at the chemical level is highly significant, not because it suggests that molecules behave in a biological fashion, but because it opens up the possibility of explaining biological behavior in chemical terms. It enables the chemical roots of that most central and profound biological theory, Darwinian theory, to be laid bare, thereby providing a truly fundamental basis for the biological - chemical connection.

\subsubsection{Chemical kinetics as the basis for Darwinian behavior}

As mentioned above, the temptation to interpret the behavior of molecular replicators in biological terms fitness, natural selection, survival of the fittest, etc., should be firmly resisted. Chemical phenomena are 
more usefully explained in chemical terms, and the competitive reactions of molecular replicators are readily addressed by the specific branch of chemistry that deals with the rates of chemical reactions - chemical kinetics. As has been appreciated since the early pioneering work of Lotka [33], the replication reaction, exemplifying an autocatalytic process, is kinetically unique in that unmitigated replication will often result in exponential growth. However, exponential growth is inherently unsustainable, so, at best, a replicator steady state would be formed in which a balance between the rates of replicator formation and replicator decay is established. This kinetic pattern can be expressed by a differential kinetic equation, such as eq 1 , where $X$ is the replicator concentration, $M$ is the concentration of building blocks from which $X$ is composed, and $k$ and $g$ are rate constants for replicator formation and decay, respectively. A steady state population, a state that is effectively 'stable', is achieved and maintained as long as $\mathrm{dX} / \mathrm{dt}$ remains close to zero. A direct consequence of this steady state description is that the stability of the resulting state is of a dynamic kind - the population of replicators is stable even though the individual members are being continually turned over.

$$
\mathrm{dX} / \mathrm{dt}=k \mathrm{MX}-g \mathrm{X}
$$

Significantly, the very existence of such dynamic states has profound chemical consequences since, as we have noted in previous work, a distinct kind of chemistry with different selection rules arises [34-36]. An example of that selection rule pointed out some years ago by Lifson [37] is the competitive reaction of two replicators competing for the same building blocks. The likely result - one of the replicators will be eliminated. Thus, at the chemical level, the competing reaction of two replicating molecules, where one of the replicators is 'driven into extinction', is a straightforward and well-understood chemical kinetic phenomenon. Given that chemistry is the more fundamental science, one can therefore say that biological natural selection emulates chemical kinetic selection, i.e., biology reduces to chemistry for this most fundamental of biological phenomena [36].

The process of complexification, the second pattern observed in both biological and chemical evolution, can also be understood as a kinetic phenomenon. It is at the chemical level, where the transformation of a simple molecular replicator to a autocatalytic network of minimal complexity can be examined directly [26-30], that the kinetic advantage of the network over the single replicator appears to manifest itself. More experimental data are needed to fully establish the connection between kinetic selection and complexification, but the preliminary evidence, particularly that provided by
Lincoln and Joyce [31], is highly suggestive. Thus kinetic selection, an inherently chemical phenomenon, one that is well-established at the molecular level, is increasingly seen to be at the root of Darwinian-type behavior, thereby providing a basis for a more fundamental understanding of Darwinian behavior at the more complex biological level.

We have identified biological 'natural selection' as an extension of chemical 'kinetic selection', but what is the chemical analogue of 'fitness', that other central Darwinian concept? What physicochemical property, if any, is being optimized by that process of chemical selection? Just where in physicochemical terms is kinetic selection leading the replicating system? In chemical processes a system is invariably driven toward a state of greater thermodynamic stability, but living systems do not seem to follow that directive as all living systems are inherently thermodynamically unstable. It turns out that the answer does lie in the system's stability, but not its thermodynamic stability, the one we normally address in chemistry. Within the replicating world there is another kind of stability, one quite distinct to thermodynamic stability, a stability kind we have termed dynamic kinetic stability (DKS) $[38,39]$. Let us briefly comment on the nature of DKS and discuss how the two kinds of stability inter-relate.

\subsubsection{Dynamic kinetic stability (DKS) and dynamic kinetic states of matter}

A system is considered stable if it is persistent, remains unchanged with time - that is an operational, phenomenological definition. Within chemical systems we recognize that a system's stability can arise for either thermodynamic or kinetic reasons and, accordingly, we speak of thermodynamic and kinetic stabilities. Importantly, both arise from lack of change. Paradoxically, however, there is another kind of stability in nature that is actually achieved through change, rather than through lack of change. This stability kind is a dynamic stability. Consider, as an example, a flowing river or a water fountain. The river or fountain, as an identifiable entity, would be classified as stable if it maintains its presence over time. That, as already mentioned, is the manifestation of stability - unchanging with time. But, of course, the water that makes up the river or fountain is changing constantly so the river's (fountain's) stability in this instance is of a dynamic kind, one that comes about through change. So though the river (fountain) as an entity is stable, its stability is of a distinctly different character to that associated with static entities.

As already discussed above, a stable population of replicating entities, whether chemical or biological, also manifests a dynamic kind of stability. The population of replicators can only be 'stable' if the individual entities 
that make up the population are continually being turned over, just like the constantly changing water content of the river or fountain. Thus one might think of the population of molecular replicators as a 'molecular fountain'. The significance of the term 'dynamic kinetic stability', as applied to a stable population of replicating entities, may now become clear. The term 'dynamic' reflects the continual turnover of the population members, the term 'kinetic' reflects the fact that the stability of the replicating system is based on kinetic parameters, such as $k$ and $g$ of eq 1 , i.e., on reaction rate constants, rather than on thermodynamic parameters. It is the values of these parameters, together with the availability of resources, which determines the stability of the particular replicating system. Accordingly we may characterize stable replicating systems (i.e., those that persist over time), whether chemical or biological, as dynamic kinetic states of matter. The utility and significance of this term can be more clearly gauged by comparison with the term frequently used to describe inanimate systems, the more traditional thermodynamic states of matter that characterize much of chemistry.

\subsubsection{The physicochemical driving force within replicator space}

Let us now specify the factors that would tend to enhance the stability of a replicating system. Fundamentally all physicochemical systems tend to undergo transformations from less stable forms to more stable forms. The second law of thermodynamics is the formal expression of that general drive. But within the constraints of the second law a range of outcomes is possible, and for reasons described above, for replicating systems, kinetic factors predominate. Specifically, within replicator space, the space in which dynamic kinetic stability is effectively in control, the selection rule becomes: from kinetically less stable to kinetically more stable. Thus, within that space the driving force is effectively the drive toward greater DKS. In other words, whereas the second law requires all chemical systems to be directed toward their most stable state (lowest Gibbs energy state), within replicator space a second law analogue effectively governs the nature of transformations [36,39]. A recent study by Boiteau and Pascal [40] also reaffirms the idea of a fundamental evolutionary driving force.

The above discussion now makes clear a major distinction between events within the physical and biological worlds. Within the physical world the second law is a useful predictor of what is likely to take place. That is how we are able to predict the melting of ice when placed in warm water, or the explosion that results from the mixing of hydrogen and oxygen gases. Generally speaking, that is the law that allows us to relate reactants and products for any reaction in an intelligible fashion. Within the biological world, however, that world of replicating systems, the second law provides effectively no predictive power. Neither the behavior of a stalking lion nor the single cell phenomenon of chemotaxis is explicable in terms of the second law. Of course all biological phenomena are consistent with the second law, but that global requirement in itself is of no predictive value. Rather, biological phenomena can be best understood and predicted on the basis of their teleonomic character $[41,42]$, a character that is totally unrelated to thermodynamic stability and the second law. The behavior of a hungry lion or of a bacterium in a glucose solution with a concentration gradient are each readily understood and predicted in teleonomic, not thermodynamic, terms. As we will discuss shortly, teleonomy, that quintessentially biological phenomenon, can be given a physicochemical basis, but it will be by relating it to kinetic, as opposed to thermodynamic, parameters.

\subsubsection{Quantification of dynamic kinetic stability}

Having established the existence of a discrete kind of stability that differs from the previously recognized stability kinds, it would be clearly beneficial to be able to quantify the concept. Unfortunately there is inherent difficulty in the formal quantification of DKS and this difficulty manifests at several levels. First, one cannot formally compare the DKS of any two arbitrary replicators, say, a bacterium and a camel, because these two entities are not directly related. In this regard the issue is not too different to thermodynamic stabilities, where one cannot formally compare the stabilities of two systems that are not isomeric. Thus, just as one cannot legitimately ask whether a molecule of water is more or less stable than a molecule of benzene, one cannot compare the relative stabilities of two replicating entities if they do not compete directly for the same material resources. Accordingly, the relative DKS of two arbitrary replicators will, in most cases, not be formally measureable.

Second, if two replicators do compete directly, as in the case of RNA oligomers competing for the same nucleotide building blocks, then the relative dynamic kinetic stabilities can be ascertained, and even quantified, based on the relative rates of replication and decay for the competing replicators. However, since DKS derives from kinetic rather than thermodynamic factors it is likely to be significantly influenced by minor variations in reaction conditions, so the significance of any particular measure would be of limited value. For example, the presence of ethidium bromide in the reaction mixture during competing RNA oligomer replication leads to a quite different competitive outcome than in its absence [43]. Accordingly, the actual magnitude of DKS for any replicating system, like its static counterpart, is highly circumstantial, and therefore is not readily amenable to meaningful quantification. In fact the difficulty in quantifying DKS is clearly reflected in attempts over many years to quantify the biological 
equivalent of DKS - 'fitness', one that began with Fisher's use of the Malthusian parameter [44]. Since that early proposal, different fitness kinds have been suggested - relative fitness, inclusive fitness, individual fitness, population fitness, and different empirical measures of that parameter have been offered, reflecting the intrinsic difficulty in quantifying the fitness concept $[45,46]$. All these different quantification proposals may in a sense be viewed as attempts to square the circle. Indeed, having reduced the biological concept of 'fitness' to the chemical concept of DKS, helps clarify the source of the difficulty by stressing the kinetic, and hence circumstantial nature of stability within a replicative context.

\subsubsection{Toward a general (extended) theory of evolution}

Once we have satisfied ourselves that the chemical and biological phases of life's emergence and evolution can be unified within a single physicochemical description, one that rests on an identifiable physicochemical driving force, the central elements of a general theory of evolution can be outlined. We begin by pointing out that the terminology employed in that formulation is necessarily physicochemical so it can address the initial phase of life's emergence, the so-called chemical phase. It follows therefore that the biological phase will also be described in physicochemical terms, but that poses no methodological difficulty - a reductionist methodology underpins much of the scientific endeavor. Accordingly the following statement will serve as the central element of the general theory:

- Certain oligomeric replicating systems, through a process of imperfect replication and on-going kinetic selection, will tend to evolve toward replicating systems of greater DKS.

While initially that process of imperfect replication might involve the preferential formation of the more rapid replicating oligomeric sequences, as demonstrated in the classic RNA replication experiments of Mills et al. [16], the emergence of replicating networks (also termed autocatalytic sets) $[2,6,47,48]$ with their enhanced replicating capability in comparison with individual molecular replicators, would open up new kinetic options within replicator space. And those particular sequences that would be capable of catalyzing the formation of other chemical classes, e.g., peptides, that exhibit catalytic activity with respect to the replication reaction itself, would further add to the process of complexification and evolution toward more stable dynamic kinetic systems. So while the process of kinetic selection between competing replicating systems can show a range of kinetic characteristics, depending on the precise replication mechanism and its particular kinetic parameters, the general trend from less complex and kinetically less stable to more complex and kinetically more stable replicators would manifest itself. Accordingly the second element of the general theory that addresses the process of complexification may be formulated as follows:

- Complexification within replicator space, through the establishment of increasingly complex chemical networks, will be the primary mechanism for the enhancement of replicator dynamic kinetic stability and the generation of stable dynamic kinetic states.

From the above discussion it becomes apparent that central Darwinian (biological) terms are just special cases of more general physicochemical terms, as indicated in Table 1. Biology, through a reductionist perspective, can be seen to merge smoothly into chemistry.

\subsubsection{Relationship between dynamic kinetic stability and} thermodynamic stability - origin and role of metabolism

Notwithstanding the above discussion and its emphasis on DKS, the relationship between that stability and thermodynamic stability needs to be clarified. After all, thermodynamic requirements, as articulated by the second law, cannot be ignored in that all transformations in the physicochemical world, regardless of whether the particular system is biological or not, must conform to its strict demands. It turns out that metabolism (in the energy-gathering sense) is the means by which Nature can have its cake and eat it. Incorporation of an energy gathering capability into the system is what enables the drive toward greater DKS to comfortably coexist with the strict requirements of the second law, despite the often opposing requirements of these two stability kinds. Let us consider this point in more detail.

Metabolism is broadly defined as the complex set of reactions that takes place in the living cell. So in that sense metabolism is the direct manifestation of the tendency toward increasing complexification that lies at the heart of the evolutionary process. However, as noted above, all chemical reactions are bound by the second law so the drive toward greater DKS and the greater complexity that frequently accompanies that stability must be consistent with the thermodynamic directive. This is true even though not all paths that seek to enhance DKS will be thermodynamically feasible. Indeed, one can presume that in many cases the greater complexity associated with enhanced DKS will actually

Table 1 Key Darwinian concepts and their underlying chemical equivalent

\begin{tabular}{ll}
\hline Biological Term & Underlying Chemical Term \\
\hline Natural selection & Kinetic selection \\
Fitness & Dynamic kinetic stability \\
Survival of the fittest & Drive toward greater dynamic kinetic stability \\
\hline
\end{tabular}


be disfavored thermodynamically, thereby effectively blocking such pathways. So how is this apparent conflict between two stability kinds resolved? The potential conflict is resolved through the emergence of a very special kind of metabolic complexification - the one specifically associated with energy gathering. It is this particular kind of metabolic capability that enables DKS and thermodynamic stability to comfortably coexist. Let us see how this can come about.

In a recent theoretical study [49], we have demonstrated that a replicating molecule that acquires an energy gathering capability through a chance mutation, e.g., through the formation of a photoactive site within the original molecule, can be expected, through a process of kinetic selection, to drive the original non-metabolic replicator into extinction. In other words, the chance emergence of a metabolic capability would lead to the formation of a replicator of greater DKS than the original non-metabolic molecule. Significantly, this result was observed even if the metabolic replicator was postulated as being inherently slower in its replicating step. Effectively the incorporation of the metabolic capability 'frees' the replicating entity from thermodynamic constraints in much the same way that a car engine 'frees' a car from gravitational constraints. A motorized vehicle is not restricted to merely rolling downhill, but through the utilization of an external energy source (gasoline), can travel uphill as well. In other words, just as a motorized vehicle is a more effective vehicle for travel, so a metabolic replicator is a more effective replicator than a non-metabolic one. The significance of the simulation described above is that it demonstrates that a metabolic capability, once acquired through a chance mutation, is likely to become incorporated into the system through a process of kinetic selection. At that point the drive toward greater DKS is no longer critically constrained by thermodynamic impediments. As we will subsequently discuss this mechanism for metabolic (energygathering) emergence has clear implications regarding the mechanism for the emergence of life. In fact that step may be considered the critical one in the transformation of a thermodynamic ('downhill') replicator into a kinetically driven, teleonomic one - the critical step that could be taken as signifying the beginning of life. Considered in this way, death is just the reversion from that (sustained) dynamic kinetic state of matter back to the traditional thermodynamic one.

\subsection{Applications of the general theory \\ 2.3.1. Explanatory power of the general theory}

Since the beginning of recorded history, man has been acutely aware of the fact that living and non-living systems are distinctly different. One key test of a general theory that attempts to encompass both animate and inanimate (as opposed to a purely biological theory) is that it should be able to address these key animateinanimate differences. Life's central characteristics that require explanation are the following:

(a) Diversity and adaptation

(b) Complexity

(c) Homochiral character

(d) Teleonomic (purposeful) character

(e) Dynamic character

(f) Far-from-equilibrium state

Of these characteristics, diversity, adaptation and complexity, seem explicable in Darwinian terms, though a recent monograph has suggested that evolutionary theory does not adequately explain diversity and complexity, and a new probabilistic principle is offered instead [50]. With regard to the remaining characteristics there is little room for argument - none have a simple Darwinian explanation. In fact Monod [41] went as far as to claim some years ago that understanding life's teleonomic character was "the central problem of biology", while Woese [1] saw in life's dynamic character an inexplicable characteristic that necessitated the abandoning of a traditional reductionist approach to the subject and to seek, as he put it, "a new biology for a new century". Let us briefly consider how each of these characteristics may be better understood in the light of the general theory.

(a) Dynamic character of living systems

As Woese [1] makes clear, living things transcend the machine metaphor: "Machines are not made of parts that continually turn over, renew. The organism is. .... Organisms are resilient patterns in a turbulent flow patterns in an energy flow". Woese clearly recognized life's dynamic nature, but was troubled that a satisfactory explanation within the traditional molecular biology framework was lacking. Let us now show how a description of life as a dynamic kinetic state of matter may go some way toward resolving Woese's dilemma.

A stable population of replicating molecules, as discussed earlier, is a dynamic state in that the population is stable even though the individual molecules are constantly turning over. Of course a dynamic population of replicating RNA molecules does not constitute life, so how does the dynamic character that we have described manifest itself in a simple life form, say a bacterial cell? For cellular replicators (say, bacteria) the dynamic character manifests itself at two levels, molecular and cellular. At the molecular level cellular proteins, a key component of all cells, are continually degraded and regenerated as part of the cell's mechanism for protein regulation [51]. As a consequence most cellular proteins have half-lives measured in hours, some even in minutes, meaning that cellular protein, a primary constituent of all living cells, is effectively completely turned over within several days, serving as yet another example of the 'molecular fountain' in operation. And, of course, at 
the cellular level, continuing turnover also takes place new cells are generated through cell division, while existing cells are constantly being degraded. Thus the dynamic character of living systems, central to their function and very existence, becomes clear through a description of life as a dynamic kinetic state of matter. Lastly, it is of interest to note that this dynamic character may also underlie multi-cellular function. For example, in the brain a significant proportion of brain cells are firing at any given moment, and consciousness, one of the most remarkable and intriguing manifestation of biological organization, has recently been attributed to highly transient groupings of neurons that are in a continuing dynamic process of change [52]. The message is increasingly clear - the dynamic nature of life's processes, at whatever level, is central to every aspect of biological function.

(b) Life's far-from-equilibrium character

The second law teaches us that systems are driven toward their lowest Gibbs energy state. Of course for kinetic reasons systems may be trapped in higher energy states for a time (e.g., a hydrogen - oxygen gas mixture), but life's far-from-equilibrium state is not simply explained in this manner. Living things continually expend energy to maintain that far-from-equilibrium state, and ion concentration gradients cannot be simply viewed as (static) kinetically stable states. In past years a feasible approach to this question was through the implementation of the theory of non-equilibrium thermodynamics [53]. That theory was able to explain how spontaneous order - so-called 'dissipative structures', can come about through the action of a perturbation on a system at equilibrium. However that approach toward biological systems ended up being increasingly questioned. The problem was that modeling living systems as dissipative structures - whirlpools, heated liquids, and the like - was unable to provide any insights into biological structure and function [54]. As was pointed out by Collier some years ago, there is no evidence that non-equilibrium thermodynamics applies to biological systems in a non-trivial manner [55]. The introduction of the DKS concept appears to resolve this dilemma. In the replicating world the stability that matters is not thermodynamic stability but DKS, of course consistent with the requirements of the second law. Thus living systems are highly stable entities despite their far-from-equilibrium character, but the stability kind is dynamic kinetic. And, as discussed above, the second law constraint is responsible for the emergence of metabolism (in the energy-gathering sense) as a critical component of all living things, enabling the two stability kinds, DKS and thermodynamic stability, to comfortably coexist.

(c) Teleonomic character

Life's teleonomic character is arguably the most striking of all of life's unique characteristics. In contrast to non-living things all life forms appear to follow an agenda. As Kauffman [2] put it: "living systems are autonomous agents - they act on their own behalf". We have recently offered a physicochemical explanation for life's teleonomic character [42], so the issue will not be discussed here in detail. Suffice it to say that its central element rests on life's description as a dynamic kinetic state of matter. Once a replicating system has taken on a metabolic (energy gathering) capability by kinetic selection (so as to enhance its dynamic kinetic stability), it is effectively 'freed' of thermodynamic constraints, and at that point the replicating system will have taken on teleonomic character. Its directive is no longer the thermodynamic directive, which defines so-called 'objective' behavior, but rather the drive toward greater DKS, whose manifestation is interpreted and understood by us as teleonomic character.

(d) Diversity

Life's diversity is clear and unambiguous. The number of species inhabiting the earth is estimated to be in the millions, occupying every conceivable ecological niche, from the poles to the equator, from the bottom of the sea to high in the atmosphere. Despite the clear evidence for diversity, the Darwinian model has provided different explanations for that diversity, from natural selection to random drift [50], the latter in line with Spencer's early concept of the "instability of the homogeneous" [56], and the topic remains a source of continuing debate [57]. In this context we would like to add the insights provided by the dynamic kinetic stability model of living systems.

One interesting distinction between the 'regular' chemical world and the replicative world lies in the different topologies of their respective spaces. As we have discussed previously [39], in the 'regular' chemical world all chemical systems are directed toward their thermodynamic sink so that the topology of that space is inherently convergent (as illustrated in 2). In contrast, within replicator space the path toward systems of greater

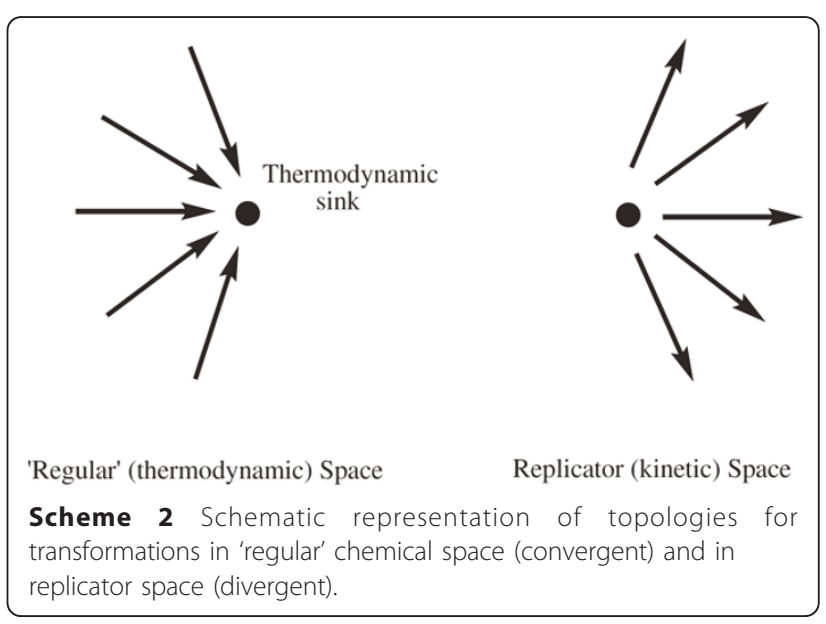


dynamic kinetic stability is not well-defined. In principle any replicating system may enhance its DKS in any number of different ways so that each system becomes a potential branching point for other kinetically stable systems, though which systems will be able to maintain that stability over time (i.e., survive), is a separate question. Accordingly, the topology of replicator space is divergent and it is this different topology that provides a simple explanation for the enormous (and constantly growing) diversity we find in the biological world. Thus Darwin's principle of divergence, a subject of continued debate [57] since it was originally proposed by Darwin, receives a simple topological explanation. This picture of convergent and divergent spaces for the two chemical worlds also explains how in the world of replicators we are able to go back in time and seek our evolutionary roots (convergent going back in time), but cannot predict future evolutionary changes (divergent going forward in time), whereas in the 'regular' chemical world we can often predict the outcome of future chemical reactions (convergent going forward in time) but are unable specify how those reacting systems came about (divergent going back in time) [39].

(e) Complexity

Much of the difficulty in explaining life's complexity is associated with the inherent thermodynamic instability associated with the organized complexity of life. Why would increasingly complex and unstable systems tend to form? However, once the nature of stability in the replicator world is clarified through the concept of DKS, the issue of complexity, at least with respect to its thermodynamic consequences, appears largely resolved. As we have already pointed out, the stability that matters in replicator space is not thermodynamic but DKS, and complexity, primarily through cross-catalytic network formation, contributes to that stability kind. We have already remarked on how two RNA enzymes were able to establish a sustained autocatalytic network, where no single enzyme on its own possessed that replicative capability [31].

An additional biological example may help to clarify the point - virus functionality. Think of a virus simplistically as a two-molecule aggregate - protein + nucleic acid. Within a biotic environment viruses are highly stable entities (in the DKS sense) in that they are able to be successfully replicated in large numbers, and thereby maintain a large population. Note, however, that the high kinetic stability arises through the cross-catalytic effect of the viral components. Each component facilitates the replication of the other, i.e., the two components are replicatively coupled. However, in that same biotic environment neither individual component will, on its own, be replicated, i.e., each individual component would manifest zero DKS. It is the system's complexity, as expressed by the cross-catalytic relationship between the viral components, which provides the means of replication, leading to the system's high DKS.

(f) Homochiral character

The stability of chiral systems in 'regular' and replicator space is strikingly different. In 'regular' chemical space a racemic mixture is inherently the more stable one; chiral excess is thermodynamically unstable, and with time all homochiral systems will tend to become transformed into the more stable racemic form (if aggregation effects are ignored). Within the replicative world, however, where kinetic factors dominate, the reverse pattern is observed. Stereochemical recognition is crucial in biological processes, particularly in the process of replication, so that in a replicative context, homochirality, which facilitates such recognition, is the preferred stereochemical outcome. In other words, due to the importance of stereochemical recognition, homochiral systems exhibit greater DKS than racemic ones. Thus the tendency of 'regular' chemical systems toward racemization, and replicating systems toward homochirality, becomes understandable in terms of the stability types within the two chemical spaces. As a final comment it is worth noting that the importance of autocatalysis is not just manifest in maintaining that homochiral dynamic kinetic state, but also in generating it. The symmetry breaking Soai reaction $[58,59]$ in which a chiral product can be formed in almost 100\% enantiomeric excess from an achiral substrate, is explicable in identical terms. Thus both the generation and the maintenance of persistent autocatalytic systems derive from the predominant influence of kinetic factors in governing those processes.

\subsubsection{Defining Life}

The only uncontroversial statement one might make regarding attempts to define life is to say the issue is highly problematic $[60,61]$. Yet a working definition is important and forms the basis for the on-going attempts to overcome at least some of the difficulties. As recently pointed out by Cleland and Chyba [60], one of the major obstacles to successfully defining life is that we are attempting to define something we don't fully understand. There are sufficient philosophic and linguistic difficulties in defining something we $d o$ understand, so the problem is only exacerbated when we attempt to define an entity whose essence remains in dispute, a source of endless debate.

Extending the evolutionary theme to inanimate systems, thereby helping to bridge the animate-inanimate gap, leads quite naturally to greater insight into what constitutes a living system. That insight, in turn, opens the door to a functional definition that may avoid at least some of the commonly recognized difficulties associated with attempts to define life in the past. 
A functional definition that seems to overcome at least some of those difficulties is as follows:

A self-sustained kinetically stable dynamic reaction network derived from the replication reaction.

Note that a central feature of the above definition is that it attempts to specify the chemical essence of life, i.e., what life is, rather than what living systems $d o$. Consider by comparison the widely cited NASA definition of life: A self-sustained chemical system capable of undergoing Darwinian evolution [62]. The fact that the NASA definition suffers from a number of deficiencies, including trivial exceptions (e.g., infertile animals, single rabbits), has been noted and discussed [60]. But the difficulty with the NASA definition appears to be more fundamental. The NASA definition is anchored in a term that is itself biological - "capable of undergoing Darwinian evolution". Ideally a life definition that strives to place living things within a general material context should be detached from its biological context. It should not contain elements that are inherently biological, as to some degree it is then defined in terms of itself.

As a final point we note that the above definition suggests that other life forms could exist, at least in principle. The general definition, here in agreement with the NASA definition, suggests that life forms not related to the protein-nucleic acid format as we know it, would be possible, and would likely exhibit the same phenomenological manifestations of the established proteinnucleic acid form that surrounds us. A detailed discussion of this issue is beyond the scope of this article.

\subsubsection{Further insights of the general theory}

Let us now point out some additional insights provided by the general theory beyond those described above. Two central questions raised in the introduction were: How did life emerge? How would we go about synthesizing a living system? What insights into these key questions does the above theory offer? Let us first address the question of life's emergence.

The proposed general theory of evolution cannot address the historical question of life's emergence from inanimate matter. Historical questions can only be addressed by uncovering the historic record and, for the early stages of abiogenesis, no historic record is available, or is likely to become available. Neither the fossil record nor phylogenetic analysis can take us back to the earliest stages of life's emergence. However, uncovering the physicochemical principles that would have facilitated such a transformation should be an achievable goal. After all, those principles would be independent of time and place and no less applicable then, as now. Indeed by viewing abiogenesis and biological evolution as one single continuous physicochemical process, we have in effect outlined the physicochemical framework that would facilitate such a transformation. In a nutshell that framework rests on the simple idea that in nature there is a special stability associated with entities that can self-replicate, a stability kind that we have termed dynamic kinetic stability. Thus autocatalysis is at the very heart of both abiogenesis and evolution. Once some relatively simple self-replicating entity would have emerged, whether a single molecule or a minimal molecular network, the drive toward greater DKS would have induced that minimal replicating system to further complexify. The precise chemical nature of that primal replicator and its precise complexification pathway, historical facts, are unlikely to be ever known. These are historic events buried deep in the mists of time, but given the centrality of the nucleic acid system as the replicating heart of all living systems, it would seem that either a nucleic acid system, or at least one closely related to nucleic acids and evolvable from it, would have been likely candidates. Importantly, however, the manner in which a thermodynamic replicator, one whose replicating reaction would have been strictly governed by thermodynamic constraints, was transformed into a farfrom-equilibrium energy-gathering teleonomic replicating system, is addressed by the theory. One might go as far as to say that the step in which a thermodynamic (down-hill) replicator was transformed into a metabolic (energy-gathering) replicator was the critical step, a saltation. That was the step, one might argue, in which a non-living chemical system began to take on the central life characteristic - teleonomic character [49], thereby crossing the threshold separating animate from inanimate.

How would we go about synthesizing a living system? We certainly are unable to provide an answer to this question, but the extended theory may provide some useful pointers, particularly as to what is unlikely to work. First, it is important to recognize that a key distinction between life and non-life is organizational, the former being a dynamic kinetic state of matter. Thus the living state is induced by the dynamic character of the biomolecules from which living things are constructed. A simplistic physical analogy that may capture that dynamic character of life is that of a juggler juggling several balls. A state where a man is standing next to those same balls is identical materially, but distinctly different organizationally. And just as it is easy to transform the juggling state to a non-juggling one (a hefty shove of the juggler is likely to do the trick), but more difficult to go in the other direction, so it is easy to transform the relatively fragile dynamic state that is life, to the static thermodynamic state representing death. So a strategy that we predict would not work would be to simply combine the molecules of life into some supramolecular aggregate. Such an aggregate would be thermodynamic in nature, not one that is 
dynamic kinetic. Based on the general model presented above, a living system could be synthesized through accessing a replicative state with a relatively simple replicating system. Once that replicative state is accessed, it could then be modified and built upon, one step at a time, ensuring the holistic replicative capability is maintained at each step. The juggler analogy is once again helpful. A juggler wanting to juggle 5 balls might start with just 2 balls and then add additional balls, one at a time - step by step. Simply tossing 5 balls at a man does not lead to a juggling state. Of course the above comments provide no practical guidance in how to achieve that desired end, and we make no pretense to suggest otherwise. Nonetheless, a general theory of evolution may provide a keener awareness of where the difficulties lie thereby helping to avoid strategies that the theory would identify as problematic.

Finally, in closing this section we briefly mention the 'metabolism first' vs. 'replication first' controversy, a long standing question at the heart of the origin of life debate $[35,63]$. In the absence of historical data that can throw light on the question of whether life began through the emergence of some replicating system which then complexified, or with the initial emergence of an autocatalytic metabolic network, a definitive resolution of the question appears unlikely. Nevertheless, having a physicochemical model that provides a basis for the transformation of inanimate to animate could provide useful insights. As the general model described above makes clear, the essence of life derives from the unique kinetic characteristics associated with autocatalysis. That, in turn, suggests that all models for the emergence of life should be analyzed with respect to that critical element. So did life begin with some primal metabolic system that was holistically autocatalytic, as proposed by the 'metabolism first' school of thought, or with some self-replicating molecule, as proposed by the 'replication first' school of thought? Consider, Joyce's work has recently demonstrated that a single RNA enzyme with its constituent building blocks is a poor replicator and is unable to bring about sustainable replication. However, the cooperative cross-catalytic system involving two RNA enzymes was able to generate a self-sustained system [31]. This key result suggests that both template-directed autocatalysis and network formation may well have been critical elements in the emergence of life, most likely closely synchronized. That being the case, we would argue that the 'replication first' - 'metabolism first' debate, as a fundamental issue in the Origin of Life debate, may no longer be of real relevance, and should be replaced with a bridging 'replication and metabolism together' scenario. Simply put, complexification (of the special kind found in biology) could not have taken place without replication, and replication without complexification had nowhere to go. The idea that the distinction between 'replication first' and 'metabolism first' schools of thought may be largely artificial was recently expressed by Eschenmoser [64].

\section{Concluding remarks}

Darwin's contribution to modern scientific thought is profound and irrevocable. It has forever changed man's view of himself and his place in the universe. By demonstrating the interconnectedness of all living things, Darwin brought a unity and coherence to biology that continues to impact on the subject to this day. But a paradoxical side product of that extraordinary contribution with its specific focus on living things, was that it resulted in a distancing between the biological and the physical sciences, one that continues to afflict the natural sciences. The disturbing result despite the enormous contribution of the Darwinian theme, Darwinism remains unable to explain what life is, how it emerged, and how living things relate to non-living ones. The challenge therefore is clear. The scientific goal - the relentless striving toward the unification of science - requires that the chasm that divides and separates the biological from the physical sciences be bridged.

In this paper we have attempted to demonstrate that by reformulating and incorporating the Darwinian theme within a general physicochemical scheme, one that rests on the concept of dynamic kinetic stability, the animate-inanimate connection can be strengthened. What the general scheme suggests is that life is, first and foremost, a highly complex dynamic network of chemical reactions that rests on an autocatalytic foundation, is driven by the kinetic power of autocatalysis, and has expanded octopus-like from some primal replicative system from which the process of complexification toward more complex systems was initiated. Thus life as it is can never be readily classified and categorized because life is more a process than a thing. In that sense Whitehead's process philosophy [65] with its emphasis on process over substance seems to have been remarkably prescient. Even the identification and classification of separate individual life forms within that ever expanding network seems increasingly problematic. The revelation that the cellular mass that we characterize as an individual human being (you, me, or the girl next door) actually consists of significantly more bacterial cells than human cells $\left(\sim 10^{14}\right.$ compared to $\sim 10^{13}$ ) [66], all working together in a symbiotic relationship to establish a dynamic kinetically stable system, is just one striking example of the difficulty. As humans we naturally focus on what we identify as the human component of that elaborate biological 
network, but that of course is an anthropocentric view, one that has afflicted human thinking for millennia. A description closer the truth would seem to be that life is a sprawling interconnected dynamic network in which some connections are tighter, others looser, but a giant dynamic network nonetheless. And it is life's dynamic character that explains why identifiable individual life forms - small segments of that giant network - can be so fragile, so easy to undermine through network deconstruction, whereas the goal of creating life is such a formidable one.

A closing remark concerning life's complexity. Life is complex - that is undeniable. But that does not necessarily mean that the life principle is complex. In fact we would argue that the life principle is in some sense relatively simple! Indeed, simple rules can lead to complex patterns, as studies in complexity have amply demonstrated $[67,68]$. So we would suggest that life, from its simple beginnings as some minimal replicating system, and following a simple rule - the drive toward greater dynamic kinetic stability within replicator space - is yet another example of that fundamental idea.

A final comment: this paper has discussed the concept of dynamic kinetic stability in some detail, and the question as to which stability kind - dynamic kinetic or thermodynamic - is inherently preferred in nature, could be asked. There is, of course, no formal answer to this question. In contrast to thermodynamic stability, dynamic kinetic stability is, as noted earlier, not readily quantifiable. Nevertheless an intriguing observation can be made. Since the emergence of life on earth from some initial replicating entity some 4 billion years ago, life has managed to dramatically diversify and multiply, having taken root in almost every conceivable ecological niche. Just the bacterial biomass on our planet alone has been estimated to be some $2 \cdot 10^{14}$ tons, sufficient to cover the earth's land surface to a depth of 1.5 meters [69]. The conclusion seems inescapable - there is a continual transformation of 'regular' matter into replicative matter (permitted by the supply of an almost endless source of energy), suggesting that in some fundamental manner replicative matter is the more 'stable' form. What implications this continuing transformation might have on cosmology in general is beyond both our understanding and the scope of this paper.

\footnotetext{
Acknowledgements

I am indebted to Prof. Jan Engberts for many valuable suggestions, to Prof. Sijbren Otto for his insightful comments on DKS quantification, and to Professors Gonen Ashkenasy, Michael Meijler, and Leo Radom for helpful comments on an earlier draft of the manuscript.
}

Received: 17 March 2011 Accepted: 7 June 2011 Published: 7 June 2011
References

1. Woese CR: A new biology for a new century. Microbiol Mol Biol Rev 2004, 68:173-186.

2. Kauffman SA: Investigations. Oxford: Oxford University Press; 2000.

3. Schrödinger $\mathrm{E}$ : What is life? The physical aspects of the living cell. Cambridge: Cambridge University Press; 1994.

4. Gleick J: Genius: The Life and Science of Richard Feynman. NY: Vintage; 1992.

5. Stankiewicz J, Eckardt LH: Chembiogenesis 2005 and systems chemistry workshop. Angew Chem Int Ed 2006, 45:342-344.

6. Ludlow RF, Otto S: Systems chemistry. Chem Soc Rev 2008, 37:101-108.

7. Dobzhansky T: Nothing in biology makes sense except in the light of evolution. The American Biology Teacher 1973, 35:125-129.

8. Haeckel E: Eine Monographie. Die Radiolarien (Rhizopoda Radiaria) Berlin: Druck und Verlag Von Georg Reimer; 1862.

9. Pereto J, Bada JF, Lazcano A: Charles Darwin and the Origin of Life. Orig Life Evol Biosphere 2009, 39:395-406.

10. Dawkins R: The blind watchmaker. New York: Norton; 1996.

11. Ruiz-Mirazo K, Luisi PL: Open questions on the origin of life. Orig Life Evol Biosphere 2010, 40:353-497.

12. Luisi PL: The emergence of life: From chemical origins to synthetic biology. Cambridge: Cambridge University Press; 2006.

13. Fry I: The emergence of life on earth. New Brunswick: Rutgers University Press; 2000.

14. Maynard Smith J, Szathmary E: The origins of life. Oxford: Oxford University Press; 1999.

15. Eigen M: Steps toward life: a perspective on evolution. Oxford: Oxford University Press; 1992.

16. Mills DR, Peterson RL, Spiegelman S: An extracellular Darwinian experiment with a self-duplicating nucleic acid molecule. Proc Natl Acad Sci USA 1967, 58:217-224.

17. Bartel DP, Szostak JW: Isolation of new ribozymes from a large pool of random sequences. Science 1993, 261:1411-1418.

18. Johnston WK, Unrau PJ, Lawrence MS, Glasner ME, Bartel DP: RNAcatalyzed RNA polymerization: Accurate and general RNA-templated primer extension. Science 2001, 292:1319-1325.

19. Joyce GF: Directed evolution of nucleic acid enzymes. Annu Rev Biochem 2004, 73:791-836.

20. Wright MC, Joyce GF: Continuous in vitro evolution of catalytic function. Science 1997, 276:614-7.

21. Voytek SB, Joyce GF: Niche partitioning in the coevolution of two distinct RNA. Proc Nat Acad Sci USA 2009, 106:7780-7785.

22. Hardin G: The competitive exclusion principle. Science 1960, 131:1292-97.

23. Sulloway FJ: Darwin and his finches: The evolution of a legend. $J$ History Biol 1982, 15:1-53.

24. Margulis L: Symbiosis in cell evolution. New Haven: Yale University Press; 1981.

25. Eigen M, Schuster $P$ : The hypercycle. A principle of natural selforganization. Berlin: Springer; 1979.

26. Sievers D, von Kiedrowski G: Self-replication of complementary nucleotide based oligomers. Nature 1994, 369:221-224.

27. Lee DH, Severin K, Yokobayashi Y, Ghadiri MR: Emergence of symbiosis in peptide self-replication through a hypercyclic network. Nature 1997 390:591-594.

28. Yao S, Ghosh I, Zutshi R, Chmielewski J: Selective amplification by autoand cross-catalysis in a replicating peptide system. Nature 1998, 396:447-450.

29. Kindermann M, Stahl I, Reimold M, Pankau WM, von Kiedrowski G: Systems chemistry: Kinetic and computational analysis of a nearly exponential organic replicator. Angew Chem Int Ed 2005, 44:6750-6755.

30. Kassianidis E, Philp D: Reciprocal template effects in a simple synthetic system. Chem Commun 2006, 4072-74.

31. Lincoln TA, Joyce GF: Self-sustained replication of an RNA enzyme. Science 2009, 323:1229-1232.

32. Weinberg S: Dreams of a Final Theory. New York: Vintage; 1994.

33. Lotka AJ: Contribution to the theory of periodic reaction. J Phys Chem 1910, 14:271-4.

34. Pross A: The driving force for life's emergence: kinetic and thermodynamic considerations. J theor Biol 2003, 220:393-406.

35. Pross A: Causation and the origin of life. Metabolism or replication first? Orig Life Evol Biosphere 2004, 34:307-321. 
36. Pross A: Seeking the chemical roots of Darwinism: Bridging between chemistry and biology. Chem Eur J 2009, 15:8374-8381.

37. Lifson S: On the crucial stages in the origin of animate matter. J Mol Evol 1997, 44:1-8.

38. Pross A, Khodorkovsky V: Extending the concept of kinetic stability: toward a paradigm for life. J Phys Org Chem 2004, 17:312-316.

39. Pross A: Stability in chemistry and biology: Life as a kinetic state of matter. Pure Appl Chem 2005, 77:1905-1921.

40. Boiteau L, Pascal R: Energy Sources, Self-organization, and the Origin of Life. Orig Life Evol Biosphere 2011, 41:23-33.

41. Monod J: Chance and Necessity. New York: Random House; 1972.

42. Pross A: How can a chemical system act purposefully? Bridging between life and non-life. J Phys Org Chem 2008, 21:724-730.

43. Saffhill $R$, Schneider-Bernloehr H, Orgel LE, Spiegelman S: In vitro selection of bacteriophage $Q \beta$ ribonucleic acid variants resistant to ethidium bromide. J Mol Biol 1970, 51:531-539.

44. Fisher RA: The Genetical Theory of Natural Selection. Oxford: Oxford University Press; 1930.

45. Demetrius L, Ziehe M: Darwinian Fitness. Theor Pop Biol 2007, 72:323-345.

46. Chevin L-M: On measuring selection in experimental evolution. Biol Letts 2011, 7:210-3.

47. Dadon Z, Wagner N, Ashkenasy G: The road to non-enzymatic molecular networks. Angew Chem Int Ed 2008, 47:6128-6136.

48. Hordijk W, Hein J, Steel M: Autocatalytic sets and the origin of life. Entropy 2010, 12:1733-1742.

49. Wagner N, Pross A, Tannenbaum E: Selective advantage of metabolic over non-metabolic replicators: a kinetic analysis. Biosystems 2009, 99:126-129.

50. McShea DW, Brandon RN: Biology's first law. Chicago: University of Chicago Press; 2010

51. Hersko A, Ciechanover A: The ubiquitin system. Annu Rev Biochem 1998, 67:425-479.

52. Greenfield S: The private life of the brain. New York: Wiley; 2000

53. Prigogine I: Time, structure and fluctuations. Science 1978, 201:777-785.

54. Peacocke AR: An introduction to the physical chemistry of biological organization. Oxford: Oxford University Press; 1989.

55. Collier J: The dynamics of biological order. In Entropy, information, and evolution. Edited by: Weber BH, Depew DJ, \& Smith JD. Cambridge MA: MIT Press; 1988:227-242.

56. Spencer H: First principles. New York: Appleton; 61900

57. Soshichi U: Darwin's Principle of Divergence.Rytro, PolandMay 26-30, 2004 [http://philsci-archive.pitt.edu/1781/1/PrDiv.pdf].

58. Soai K, Shibata T, Morioka H, Choji K: Asymmetric autocatalysis and amplification of enantiomeric excess of a chiral molecule. Nature 1995, 378:767-768.

59. Soai K, Kawasaki T: Asymmetric autocatalysis with amplification of chirality. Top Curr Chem 2008, 284:1-33.

60. Cleland CE, Chyba CF: Defining life. Orig Life Evol Biosphere 2002, 32:387-393.

61. Lazcano A: What Is Life? A brief historical overview. Chem \& Biodiversity 2008, 5:1-15.

62. Joyce GF: Foreword. In Origins of life: the central concepts. Edited by: Deamer DW, Fleischaker GR. Boston: Jones 1994:

63. Shapiro R: Small molecule interactions were central to the origin of life. Quart Rev Biol 2006, 81:105-125.

64. Eschenmoser A: Question 1: Commentary referring to the statement: The origin of life can be traced back to the origin of kinetic control and the question do you agree with this statement? Orig Life Evol Biosphere 2007, 37:309-314.

65. Whitehead AN: Process and Reality: An Essay in Cosmology. New York: Free Press; 1978

66. Berg R: The indigenous gastrointestinal microflora. Trends in Microbiology 1996, 4:430-435.

67. Wolfram S: A new kind of science. Champaign IL: Wolfram media; 2002

68. Weinberg S: Is the universe a computer? NY Rev Books 2002, 49:43-47.

69. Gold T: The deep, hot biosphere. Proc Nat Acad Sci USA 1992, 89:6045-49.

doi:10.1186/1759-2208-2-1

Cite this article as: Pross: Toward a general theory of evolution:

Extending Darwinian theory to inanimate matter. Journal of Systems

Chemistry 2011 2:1.

\section{Publish with ChemistryCentral and every scientist can read your work free of charge \\ "Open access provides opportunities to our colleagues in other parts of the globe, by allowing anyone to view the content free of charge." \\ W. Jeffery Hurst, The Hershey Company.}

- available free of charge to the entire scientific community

- peer reviewed and published immediately upon acceptance

- cited in PubMed and archived on PubMed Central

- yours - you keep the copyright

Submit your manuscript here:

http://www.chemistrycentral.com/manuscript/<smiles>c1ccccc1</smiles>

Chemistry Centra 